\title{
The Transition Between Dripping and Jetting of a Liquid Jet
}

\author{
Chun-Kuei CHEN ${ }^{1 *}$, Cheng-Yao TSAI ${ }^{2}$, Ta-Hui LIN ${ }^{1,2^{*}}$ \\ ${ }^{1}$ Research Center for Energy Technology and Strategy, National Cheng Kung University, \\ Taiwan \\ ${ }^{2}$ Department of Mechanical Engineering, National Cheng Kung University, Taiwan \\ *Corresponding author email : c_k_chen@mail.ncku.edu.tw
}

\begin{abstract}
The objective of this research is to analyze the characteristics of a liquid jet under different working fluid properties and flow rates. In this study, glycerol-water mixtures with different concentrations $(\Omega)$ were used as the working fluids. The properties of the working fluid were varied in terms of the main viscosity $(\mu)$, the diameter of the liquid column $\left(d_{j}\right)$, and the flow rate $(\dot{Q})$ to investigate the characteristics of the breakup length $\left(L_{j}\right)$ and the flow type transition between a dripping and jetting flow. From the research results, when the $\Omega$ were increased the $L_{j}$ and $D$ extended and increased, respectively. Hysteresis behavior occurred in the liquid column with a larger diameter of $(\geq 1146 \mu \mathrm{m})$, which indicated that the critical flow rate of the transition from dripping to jetting $(D J)$ was different from that from jetting to dripping $(J D)$. The range of the hysteresis increased as the $\Omega$ increased and moved to a lower We number region, especially with a larger $d_{j}$. It was found that increases in $\Omega$ also caused hysteresis behavior to occur in $d_{j}$ that without hysteresis.
\end{abstract}

\section{Keywords}

Breakup Length, Rayleigh Instability, Hysteresis, Fluid Properties, Dripping \& Jetting.

\section{Introduction}

The efficiency of technical equipment has jumped to a new level since the industrial revolution in the 18th century. Since then, many researchers have systematically studied various areas of science, such as the combustion process, control engineering, material science, and so forth. As a result, human beings have gained the right to use the resources provided by the power of knowledge.

Spray system technology plays a very important role in current industrial applications. Based on the surface properties of a given material, the impingement study of droplets can determine whether the surface of a material is hydrophobic or not; on the application side of combustion technology, the atomization characteristics and droplet distribution grouping generated by a nozzle will also affect engine efficiency. In addition to industrial applications, uniform droplet generator [1] technology is also widely used in medicine, food processing [2], and electronic printing [3]. Therefore, there are many studies focusing on the mechanism of fluid atomization, breakup patterns, droplet formation, etc.

There have been a lot of investigations on dripping flows (periodic dripping, dripping faucet) from all perspectives but the transition of flow type has not appeared to attract much attention. When the flow type is going to transform, there exists a condition where the fluid velocity must reach a specific value. Tyler \& Richardson [4] were the first scientists to conduct numerous experiments intended to identify the transition from dripping to jetting. They also proposed the threshold velocity. However, the phenomenon of flow type transition was not fully explored until the work published by Clanet \& Lasheras [5] and Hoeve van et al. [6]. Basaran et al. [7] attempted to determine the transition point between periodic dripping (PD), the dripping faucet (DF), and jetting. Based on their experiment and simulation results, there 
will be no chaotic dripping when $O h>0.5$, where the range of the complex dripping behavior became wider if $\mathrm{O}$ decreased.

Ambravaneswaran et al. [8] and Subramani et al. [9] conducted experimental and simulation analyses to provide a criterion for the transition to occur at a critical We number, which was shown to be a function of the Bo of the inside and outside diameters of a tube and they empirically obtained the constant $K$ with a value of 0.372 . In their research, not only water but also a high viscosity liquid, e.g., syrup, was used as the working fluid. The results showed that high viscosity liquids transition directly from simple dripping to jetting as the flow rate increases if the $B o$ and $O h$ numbers are sufficiently high. In addition, high viscosity liquids may eliminate all the complex nonlinear dynamic responses exhibited by low viscosity liquids.

While a large number of scientists are committed to predicting the threshold of the flow type transition, the hysteresis phenomenon has occurred in many types of research [5, 8-12]. Hysteresis is used to elaborate on the critical flow rate corresponding to the transition from dripping to jetting and is different from the critical flow rate from jetting to dripping. As shown in Figure 1 when the flow rate continuously increases, which is from dripping to jetting $(D J)$, the flow type translates to jetting until $\dot{Q}=0.9 \mathrm{ml} / \mathrm{s}$; conversely, when the flow rate continuously decreases, which is from jetting to dripping $(J D)$, instead, the flow type translates to dripping occurring at $\dot{Q}=0.7 \mathrm{ml} / \mathrm{s}$. The interval, which in this case is $\dot{Q}=0.75-0.85 \mathrm{ml} / \mathrm{s}$, is the hysteresis region. However, hysteresis behavior is always mentioned but rarely investigated.

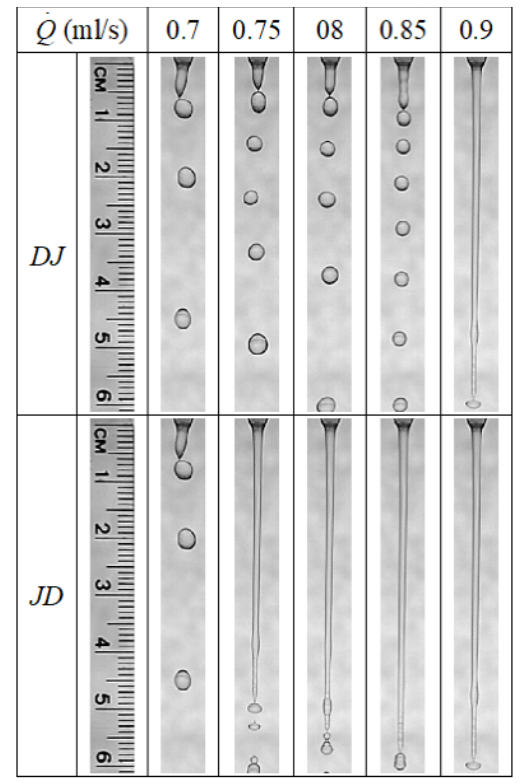

Figure 1. Demonstration of hysteresis behaviour

Previously in our research team, Chang [13] proceeded to experimentally analyze the breakup behavior of a rotating liquid column $(R L C)$ and vigorously surveyed the transition point of the relationship between dripping and jetting for an RLC. Based on Chang's [13] results, the JD transition point will be affected by the imposed rotation, where the critical rotating speed is linearly proportionate to the flow rate; however, there is no obvious change in the DJ transition. Similarly, hysteresis behavior also occurs near the flow type transition point, but this is only a preliminary explanation.

Therefore, hysteresis behavior is more deeply investigated in this study, where the properties of the working fluid are varied in order to explore their effect on hysteresis behavior. In addition, the issue of the lack of hysteresis behavior in smaller nozzles in Chang's [13] study also triggered a new investigation. 


\section{Material and Methods}

A schematic of the experimental setup is shown in Figure 2. The working fluid reservoir is fixed on the top of an extendable steel rod, which makes it easier to control the flow rates in the entire experimental system. The reservoir is connected to the upper part of the liquid nozzle with a plastic tube. After the fluid exits the plastic tube, the fluid accumulates in the chamber of the liquid nozzle. The nozzle system is divided into two parts: The upper part is the non-rotating chamber used for fixing the whole system; the lower part is the rotating mechanism. The rotating mechanism is composed of two parts. The first part is made up of a roller bearing and two joint shaft pins on both ends. The top end is obviously connected to the non-rotating chamber, allowing the fluid to flow by, whereas the opposite end is connected to the second part of the rotating machinery.

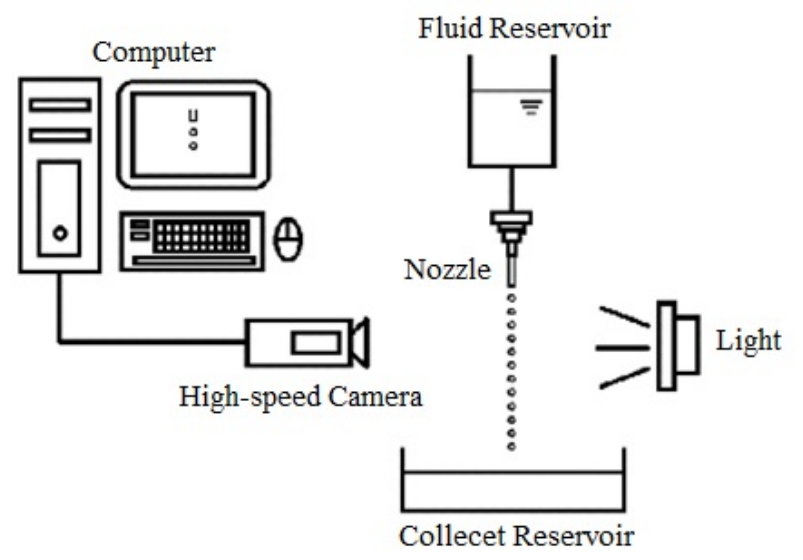

Figure 2. Experimental apparatus

In this study, we use glycerol-water mixtures as the working fluid and vary the weight ratio of glycerol in the mixture $(\Omega)$. The main parameter we concern about is viscosity $(\mu)$ and there are no obvious change in the density $(\rho)$ and the surface tension $(\sigma)$ as $\Omega$ increases. Table 1 shows the properties of working fluid at five different concentrations. The experimental parameters are listed in Table 2, which shows the range of the nozzle diameters $\left(d_{j}\right)$, flow rates $(\dot{Q})$, and concentrations of the working fluid $(\Omega)$.

Table 1 - Properties of glycerol-water mixtures

\begin{tabular}{cccc}
\hline$\Omega(\mathrm{wt} \%)$ & $\rho\left(\mathrm{kg} / \mathrm{m}^{3}\right)$ & $\mu\left(\mathrm{N} \cdot \mathrm{s} / \mathrm{m}^{2}\right)$ & $\sigma(\mathrm{N} / \mathrm{m})$ \\
\hline 0 & 998 & $1.04 \times 10^{-3}$ & $73.2 \times 10^{-3}$ \\
\hline 10 & 1027.4 & $1.38 \times 10^{-3}$ & $72.45 \times 10^{-3}$ \\
\hline 20 & 1047 & $1.84 \times 10^{-3}$ & $71.7 \times 10^{-3}$ \\
\hline 30 & 1086 & $2.97 \times 10^{-3}$ & $70.85 \times 10^{-3}$ \\
\hline 40 & 1100 & $3.63 \times 10^{-3}$ & $70 \times 10^{-3}$ \\
\hline
\end{tabular}

Table 2 - Experimental parameters

\begin{tabular}{ccc}
\hline$d_{j}(\mu \mathrm{m})$ & $\dot{Q}(\mathrm{ml} / \mathrm{s})$ & $\Omega(\mathrm{wt} \%)$ \\
\hline $582 \sim 1500$ & $0.2 \sim 0.9$ & $0 \sim 40$ \\
\hline
\end{tabular}




\section{Results and Discussion}

Figure 3 (a) to (e) show the relationship between the breakup length and flow rates under different concentrations of glycerol-water mixtures, $\Omega$, where the left-hand side is the outcome with smaller nozzles, and the right hand-side is the outcome with larger nozzles. From these figures, it is obvious that when the flow rate increases, the breakup length becomes longer, the breakup length and flow rate are linearly proportional. The structure of the liquid column becomes stronger because of the enhanced inertial force in the gravitation direction caused by the increase in the flow rate. Thinking from the perspective of the mass flow rate, the jet velocity and the cross-section area of the nozzle are inversely proportional, which is why if the cross-section area is smaller, the jet velocity is faster. Thus, at the same flow rate, the breakup length will decrease as the nozzle diameter, $d_{j}$, increases.

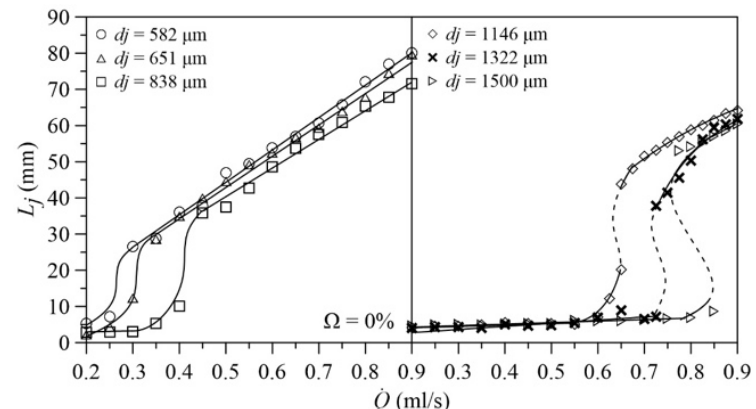

(a)

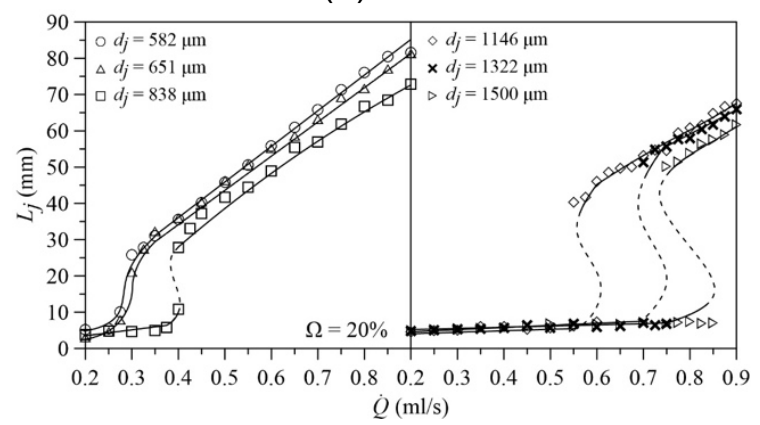

(c)

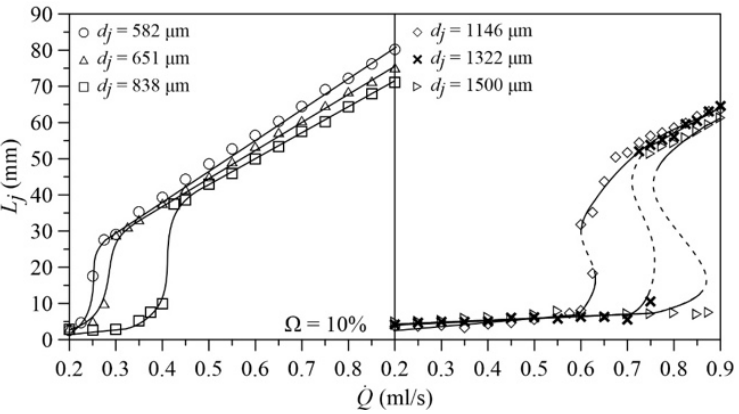

(b)

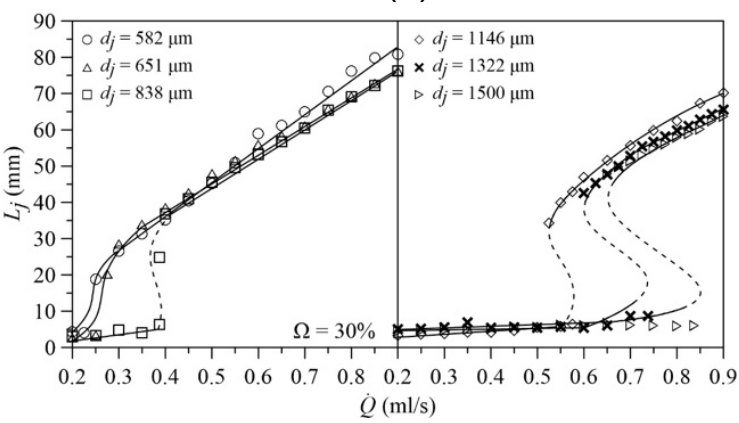

(d)

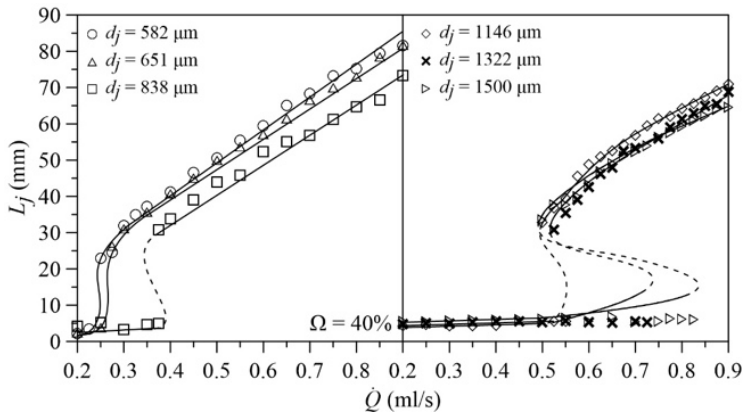

(e)

Figure 3. Breakup length of jets with different $\dot{Q}$ and $d_{j}$ for various $\Omega$

Furthermore, Figure 4 (a) to (e) shows the relationship between breakup length and jet velocity. Excluding the effect of the cross-section area of the nozzle and only considering the effect of jet velocity on the breakup length does lead to a completely different conclusion. Different from the results shown in Figure 3 (a) to (e), at the same jet velocity, the outcome of breakup length for the larger nozzles is longer than it is for smaller nozzles because more inertial force is needed to drive the liquid column from larger nozzles to reach the same jet velocity. In addition, from Rayleigh's [[13]] model, in order to cause the liquid column to become unstable, the wavelength of an axisymmetric perturbation is necessarily greater than 
the perimeter of the liquid column $\lambda \geqq \pi d_{j}$, and the amplitude of perturbation is equal to $r_{j}$; that is to say, it takes more time to cause the larger liquid column to break up. Hence, this leads to the breakup length of the larger nozzles being longer at the same jet velocity.

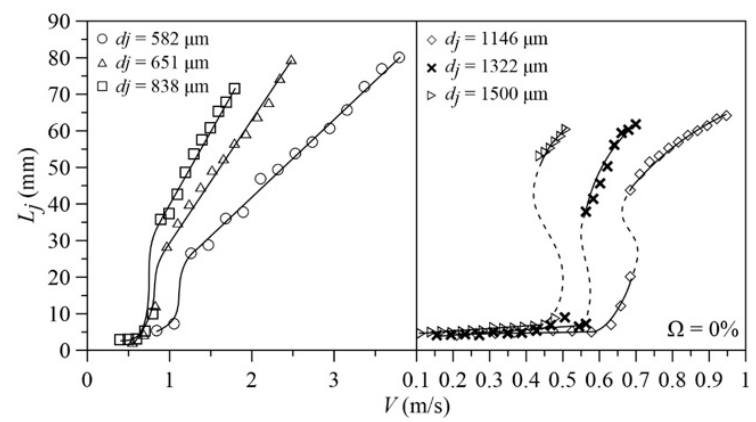

(a)

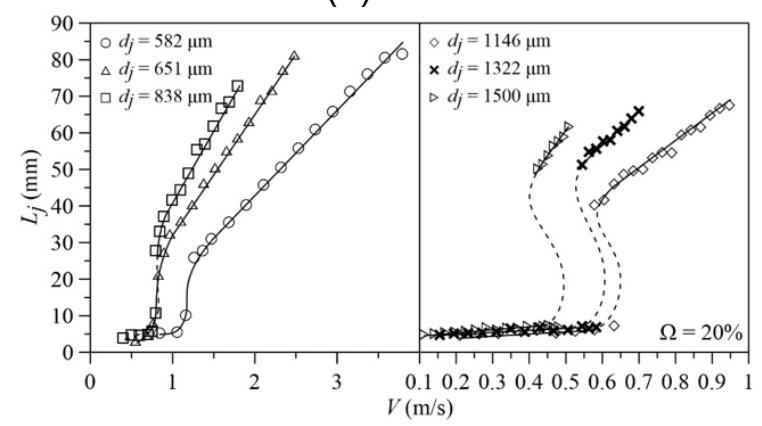

(c)

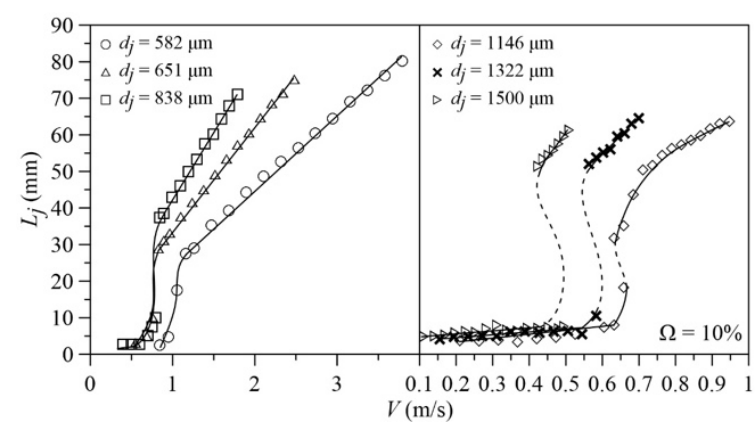

(b)

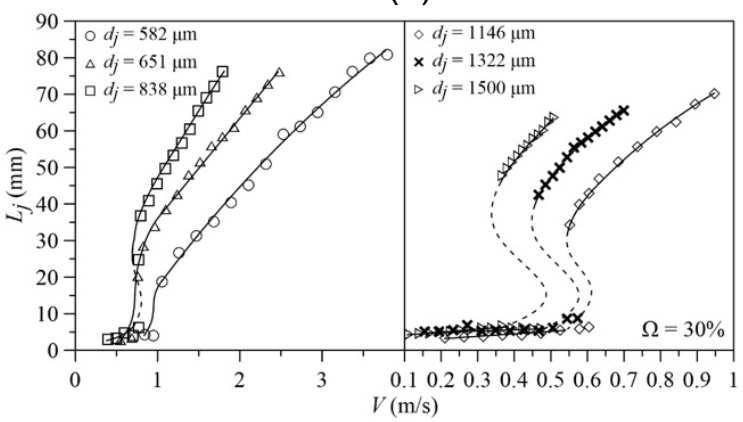

(d)

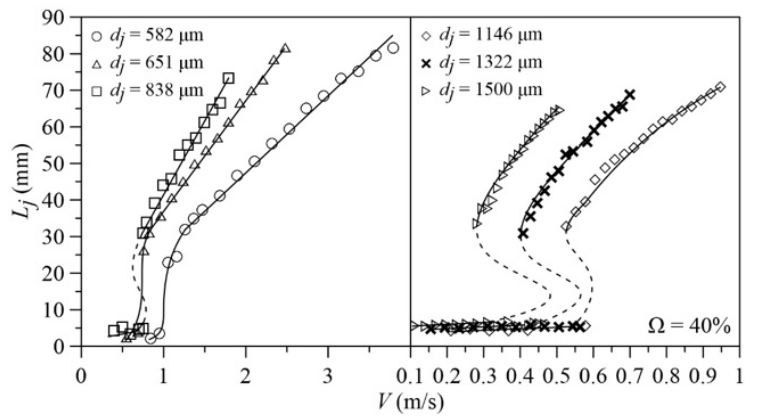

(e)

Figure 4. Breakup length of jets with different $V$ and $d_{j}$ for various $\Omega$

Figure 5 and Figure 6 show the breakup length as functions of $d_{j}, \Omega$ and $\dot{Q} / V$, respectively, where the effects of the properties of the working fluid on the breakup length for each nozzle can also be observed. In this research, when we vary the properties of the working fluid, compared to surface tension and density, variations in viscosity are significant; thus the effect of viscosity is the main factor of interest. From Figure $\mathbf{5}$ and Figure $\mathbf{6}$, it is easy to observe that the breakup length will increase as the $\Omega$ increases at the same nozzle diameter. Because the increase of viscosity will cause a damping effect to occur, and the perturbation caused by interaction with surrounding air will be damped out. Therefore, the damping effect makes it difficult for the liquid column to break and thus leads to a longer break length. 


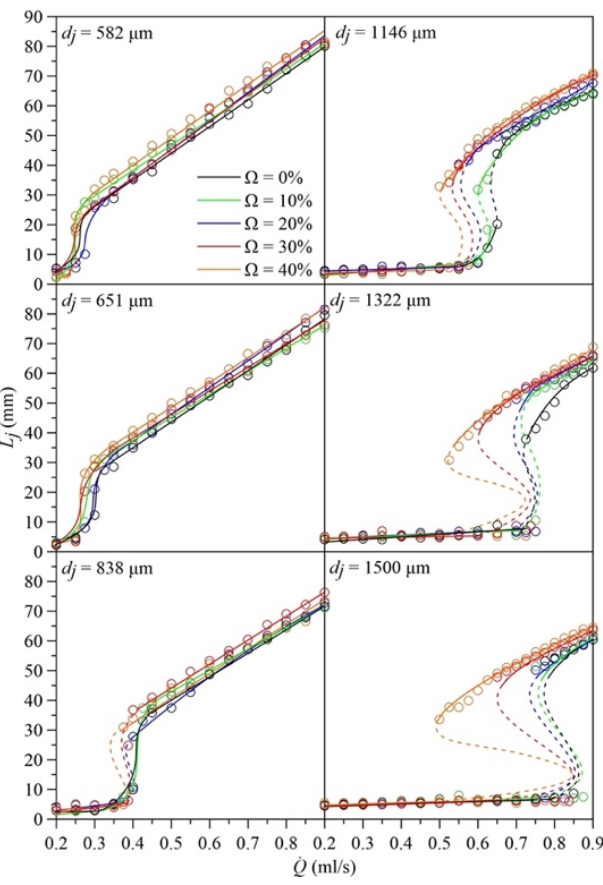

Figure 5. Breakup length as functions of $\dot{Q}, d_{j}$ and $\Omega$

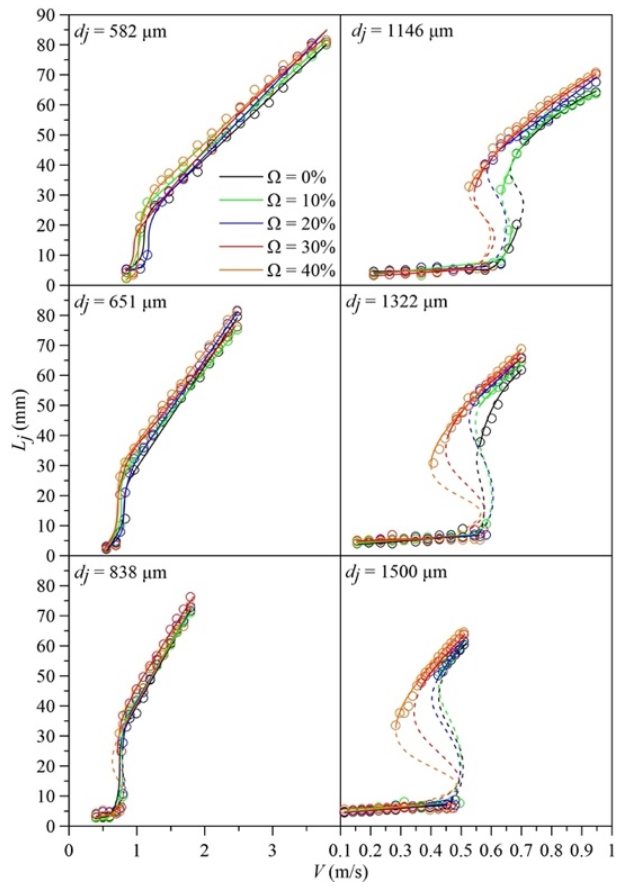

Figure 6. Breakup length as functions of $V, d_{j}$ and $\Omega$

Although the empirical correlations for predicting the breakup length of a laminar jet proposed by Rayleigh [14] and Weber [15] lay a good foundation, the model they proposed is too ideal to be put into a realistic model even though the effect of viscosity was already taken into account in Weber's [[14]] model. However, the empirical correlation suggested by Grant \& Middleman [16] is plotted as a red line in Figure 7 (a) to (e), where the empirical correlation can be expressed as:

$$
L_{j} / d_{j}=19.5\left(W e^{0.5}+3 W e / R e\right)^{0.85}
$$

Figure 7 (a) to (e) shows the non-dimensional breakup length varying with We number for different $\Omega$, where the predictions given by Eq. (1) are in good agreement with the experimental data except in regions where the flow rate is relatively low. For example, in Figure 7 (a), when the We number decreases below 10, the flow type begins to become a dripping flow for all smaller nozzles, and the average error between the experimental data and the predictions is around 2.5 times. Thus, the empirical correlations are always used only for predicting the values under a jetting flow.

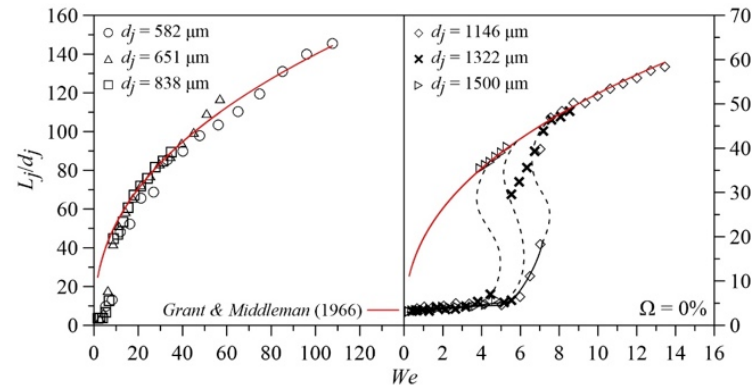

(a)

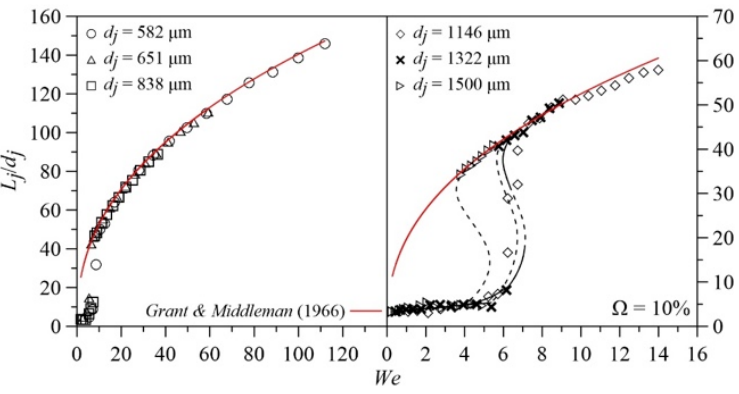

(b) 


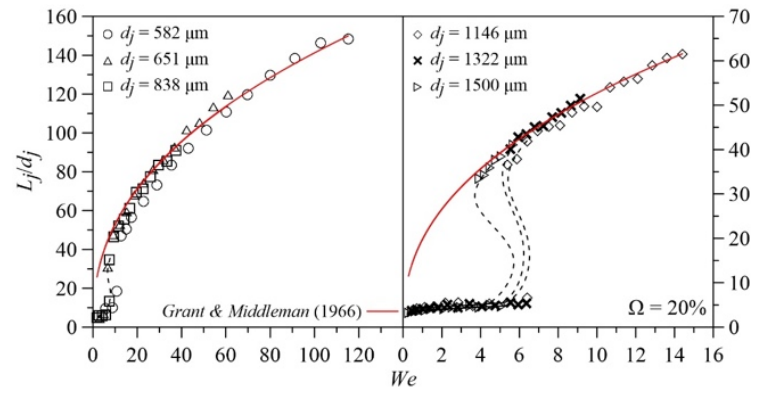

(c)

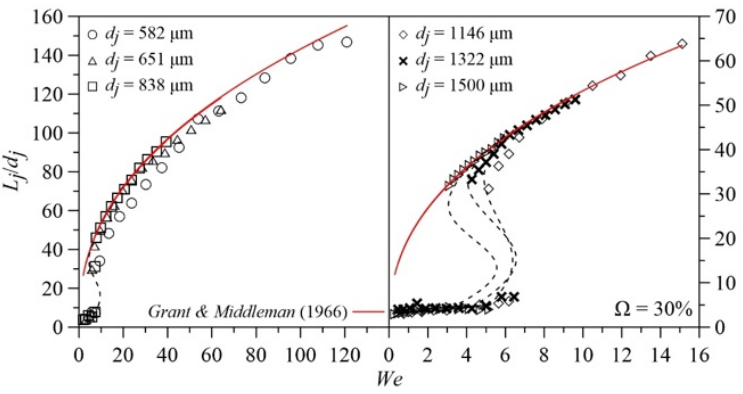

(d)

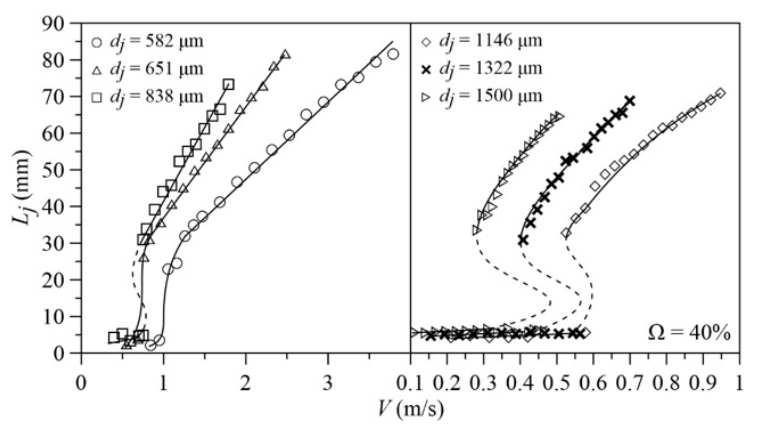

(e)

Figure 7. Non-dimensional breakup length of jets with different $d_{j}$ for various $\Omega$

\section{Conclusions}

The variations in $\Omega$ were also found to have a significant effect on the transition points from jetting to dripping $(J D)$ in the case of the larger nozzles, $d_{j}=1146,1322,1500 \mu \mathrm{m}$. Because the bonding between the working fluid molecules becomes stronger when $\Omega$ is increased, there is still a jetting flow in spite of the lower We number. Also, the hysteresis region extends and moves to a lower We number with increases in $\Omega$, where a larger nozzle causes this phenomenon to be more significant. In addition, nozzle geometry was utilized to explain why hysteresis behavior will not occur in smaller nozzles, where $d_{j}=582,651,838 \mu \mathrm{m}$. Because the ratios (thickness / inner radius) of smaller nozzles are larger; that is, the inertial force is less than the surface tension caused by the wetting area, it is difficult to maintain the jetting flow near the transition point. Interestingly, because the ratio of $d_{j}=838 \mu \mathrm{m}$ is closer to that of larger nozzles, once the $\Omega \geq 20 \%$, hysteresis will occur. The variation of the hysteresis region is still very much in the experimental stage and warrants further investigation. Much more also needs to be known about the influence of working fluid properties on the hysteresis region.

\section{Acknowledgments}

This work was supported by the Ministry of Science and Technology, Taiwan, under contract of MOST 107-2218-E-006-023 -.

\section{Nomenclature}
Bo Bond number
$D \quad$ Breakup droplet size $[\mu \mathrm{m}]$
$d_{j} \quad$ Diameter of the liquid column $[\mu \mathrm{m}]$
$K$ Wavenumber
$L_{j} \quad$ Breakup length of a jet [mm]
Oh Ohnesorge number 
$\dot{Q} \quad$ Flow rate $[\mathrm{ml} / \mathrm{s}]$

Re Reynold number

$\checkmark \quad$ Jet velocity $[\mathrm{m} / \mathrm{s}]$

$r_{j} \quad$ Normally refers to nozzle radius $[\mu \mathrm{m}]$

We Weber number

$\lambda \quad$ Wavelength of a jet

$\mu \quad$ Viscosity $\left[\mathrm{N} \cdot \mathrm{s} / \mathrm{m}^{2}\right]$

$\rho \quad$ Density $\left[\mathrm{kg} / \mathrm{m}^{3}\right]$

$\sigma \quad$ Surface tension $[\mathrm{N} / \mathrm{m}]$

$\Omega \quad$ Weight ratio of glycerol in glycerol-water mixture [wt\%]

\section{References}

[1] Jiang, X. S., Qi, L. H., Luo, J., Huang, H., Zhou, J. M., 2010, The International Joumal of Advanced Manufacturing Technology, 49, pp. 535-541.

[2] Berkland, C., Kim, K., Pack, D. W., 2001, Joumal of Controlled Release, 73, pp. 59-74.

[3] Xu, C., Chai, W., Huang, Y., 2015, Biotechnology and Bioengineering, 109, pp. 3152-3160.

[4] Tyler, E., and Richardson, E. G., 1925, Proceedings of the Physical Society of London (1874-1925), 37, pp. 297.

[5] Clanet, C., and Lasheras, J. C., 1999, Joumal of Fluid Mechanics, 383, pp. 307-326.

[6] Hoeve, W., Gekle, S., Snoeijer, J. H., Versluis, M., Brenner, M.P., Lohse, D., 2010, Physics of Fluids, 22, 122003.

[7] Basaran, O. A., 2002, AIChE Joumal, 48, pp. 1842.

[8] Ambravaneswaran, B., Subramani, H. J., Phillips, S. D., Basaran, O. A., 2004, Physical Review Letters, 93, $0345011-0345014$.

[9] Subramani, H. J., Yeoh, H. K., Suryo, R., Xu, Q., Ambravaneswaran, B., Basaran, O. A., 2006, Physics of Fluids, 18, 03210613.

[10] Sartorelli, J. C., Gonçalves, W. M., Pinto, R. D., 1994, Physical Review E, 49, 3963.

[11] Coullet, P., Mahadevan, L., Riera, C. S., 2005, Joumal of Fluid Mechanics, 526, pp. 1-17.

[12] Mariano, R. R., Paloma, T., Alejandro, S., 2018, International Joumal of Multiphase Flow, 104, pp. 206-213.

[13] Chang, C., C., "A Study on the Breakup of a Rotating Liquid Jet," Unpublished Master Dissertation, Department of Mechanical Engineering National Cheng Kung University, 2018.

[14] Lord Rayleigh, 1879, Proceedings of the Royal Society of London, 29, pp. 71.

[15] Weber, C., 1931, ZAMM , 11, pp. 136-154.

[16] Grant, R. P., Middleman, S., 1966, AIChE Joumal, 12, pp. 669-678. 